\title{
Semi-Lagrangian Implicit-Explicit Two-Time-Level Scheme for Numerical Weather Prediction
}

\author{
Andrei Bourchtein \\ Mathematics Department, Pelotas State University, Brazil \\ bursteinaterra.com.br
}

\begin{abstract}
Semi-Lagrangian two-time-level finite difference scheme for hydrostatic atmospheric model is considered. Approximation of the gravitational waves in implicit-explicit manner allows to keep balance between extended stability and required accuracy. Both are assured by implicit discretization of the fast principal vertical modes and explicit approximation of the slow secondary gravitational waves. Numerical experiments with actual atmospheric data are carried out to define the most efficient implicit-explicit separation, which produces the accurate forecasts at the less computational cost.
\end{abstract}

\section{Introduction}

Semi-Lagrangian (SL) approach has been proved to be an efficient alternative to Eulerian one because it allows to circumvent the Courant-Friedrichs-Lewy (CFL) criterion related to advection velocity, requiring only solution of trajectory equations, which represent the systems of ODE decoupled at each grid point and solved efficiently by iterative algorithm [7,15]. If, additionally, gravitational terms of hydrostatic atmospheric model are discretized with sufficient degree of implicitness, then time step of SL schemes can be chosen on the base of accuracy considerations $[7,15]$. This is great advantage of such schemes because more straight explicit and implicit discretizations are computationally expensive: explicit approximation requires very small time steps due to fast gravitational waves and implicit approximation requires solution of nonlinear PDEs at each time step.

The choice of the level of implicitness of gravitational terms in SL schemes can be based on considerations of efficiency, including the accuracy of forecasting fields and minimization of computational cost. The most direct approximation is implicit, which is used in the majority of the SL schemes [7,15]. It allows to use a great time steps, but it is not the most efficient way because the 3D linear algebraic systems of the high order $M \times N \times L$ (where $M, N$ and $L$ are the number of points in horizontal and vertical directions) should be solved at each time step. There is a chance to avoid these hard computations by separating the spectrum of gravitational waves. It is well known that highest internal barotropic modes of the vertically decoupled hydrostatic equations contain the gravitational waves with the smallest amplitudes and slowest velocities of propagation [8]. Such waves are secondary and do not impose any relevant restriction on time step and, consequently, they can be discretized in explicit 
manner on a coarse grid. This way, implicit part can be reduced to $K$ separate 2D linear systems of the order $M \times N$, where $K$ is a small as compared to $L$. Thus, the computational cost can be reduced if the slow gravitational waves are discretized more explicitly and coarsely.

A similar approach has been used in a three-time-level SL model [3]. The reported results showed strong points of developed scheme for time steps up to $40 \mathrm{~min}$. In the last decade, motivated by results of McDonald [10] and Temperton and Staniforth [16] for shallow water equations, numerical modelers started to substitute tree-timelevel SL schemes by two-time-level ones, which allow to achieve the same accuracy with even larger time steps $[9,11,17]$. In this paper we apply the modified technique of [3] to two-time-level scheme with objective to increase time step up to $60 \mathrm{~min}$ with no loss of forecast accuracy.

\section{Semi-Lagrangian Implicit-Explicit Time Discretization}

Primitive equations in time coordinate $t$, horizontal Cartesian coordinates $x, y$ and vertical coordinate $\sigma=p / p_{s}$ can be written as follows [7]:

$$
\begin{gathered}
d_{t} u=f_{0} v-G_{x}+N_{u}, d_{t} v=-f_{0} u-G_{y}+N_{v}, \\
G_{\ln \sigma}=-R T, d_{t} P=-D-\dot{\sigma}_{\sigma}, c_{p} d_{t} T=R T_{0} \cdot\left(d_{t} P+\dot{\sigma} / \sigma\right)+c_{p} N_{T} ; \\
N_{u}=\left(f-f_{0}\right) v-R\left(T-T_{0}\right) P_{x}, N_{v}=-\left(f-f_{0}\right) u-R\left(T-T_{0}\right) P_{y}, \\
c_{p} N_{T}=-R\left(T-T_{0}\right)\left(\dot{\sigma} / \sigma-D-\dot{\sigma}_{\sigma}\right) .
\end{gathered}
$$

Here, $u, v$ and $\dot{\sigma}$ are horizontal and vertical velocity components, $D=u_{x}+v_{y}$ is the horizontal divergence, $P=\ln p_{s}, p$ and $p_{s}$ are the pressure and surface pressure respectively, $T$ is the temperature, $G=g z+R T_{0} P, z$ is the height. Nonlinear and variable coefficient terms are grouped in $N_{u}, N_{v}, N_{T}$. Individual 3D derivative is

$$
d_{t} \varphi=\varphi_{t}+u \varphi_{x}+v \varphi_{y}+\dot{\sigma} \varphi_{\sigma} \quad, \varphi=u, v, P, T
$$

and the following parameters are used: $f$ is the Coriolis parameter with the mean value $f_{0}, g$ is the gravitational acceleration, $R$ is the gas constant of dry air, $c_{p}$ is the specific heat at constant pressure, $T_{0}=$ const is the reference temperature profile. Hereinafter the subscripts $t, x, y, \sigma$ denote the partial derivatives with respect to indicated variable.

Let us split solution of (1)-(2) into two steps. The first SL step consists of solution of the advective part of the prognostic equations

$$
d_{t} \mathbf{r}=\mathbf{V}, \mathbf{r}=(x, y, \sigma), \mathbf{V}=(u, v, \dot{\sigma}) .
$$

These equations are efficiently solved by Robert's iterative algorithm $[14,15]$, which assures the second order of accuracy and converges under limitation on time step expressed in the terms of the wind derivatives $[13,15]$ : 


$$
\tau \leq 2 / 3 V_{d}, \quad V_{d}=\max \left(\left|u_{x}\right|,\left|u_{y}\right|,\left|u_{\sigma}\right|,\left|v_{x}\right|,\left|v_{y}\right|,\left|v_{\sigma}\right|,\left|\dot{\sigma}_{x}\right|,\left|\dot{\sigma}_{y}\right|,\left|\dot{\sigma}_{\sigma}\right|\right),
$$

For fine grid with horizontal meshsize about $50 \mathrm{~km}$ and 20 vertical levels it gives maximum time step about 1 hour.

The other terms, including gravitational waves, are considered on the second step

$$
\begin{gathered}
u_{t}=f_{0} v-G_{x}+N_{u}, v_{t}=-f_{0} u-G_{y}+N_{v}, \\
P_{t}=-D-\dot{\sigma}_{\sigma}, c_{p} T_{t}=R T_{0} \cdot\left(P_{t}+\dot{\sigma} / \sigma\right)+N_{T} .
\end{gathered}
$$

Implicit discretization of all the gravitational terms $\left(G_{x}, G_{y}, D, \dot{\sigma}_{\sigma}, \dot{\sigma} / \sigma\right)$ is the most traditional approximation in SL models $[7,15,17]$. It gives rise to the following time difference equations:

$$
\begin{gathered}
\frac{u^{n+1}-u^{n}}{\tau}=f_{0} \frac{v^{n+1}+v^{n}}{2}-\frac{G_{x}^{n+1}+G_{x}^{n}}{2}+N_{u}^{n+1 / 2}, \\
\frac{v^{n+1}-v^{n}}{\tau}=-f_{0} \frac{u^{n+1}+u^{n}}{2}-\frac{G_{y}^{n+1}+G_{y}^{n}}{2}+N_{v}^{n+1 / 2} \\
\frac{P^{n+1}-P^{n}}{\tau}=-\frac{D^{n+1}+D^{n}}{2}-\frac{\dot{\sigma}_{\sigma}^{n+1}+\dot{\sigma}_{\sigma}^{n}}{2}, \frac{T^{n+1}-T^{n}}{\tau}=\frac{R T_{0}}{c_{p}}\left(\frac{P^{n+1}-P^{n}}{\tau}+\frac{\dot{\sigma}^{n+1}+\dot{\sigma}^{n}}{2 \sigma}\right)+N_{T}^{n+1 / 2},
\end{gathered}
$$

where $\tau$ is the time step, superscript $n+1$ denotes the values at the new time level $t_{n+1}=(n+1) \tau$ and superscript $n$ denotes the values at the current time level $t_{n}=n \tau$. The nonlinear terms are evaluated by extrapolation to the half way time level $t_{n+1 / 2}$ :

$$
N^{n+1 / 2}=\left(3 N^{n}-N^{n-1}\right) / 2, N=N_{u}, N_{v}, N_{T} .
$$

The scheme (7)-(9) is of the second order of accuracy and linear analysis shows that it is absolutely stable. However, it requires the solution of $3 \mathrm{D}$ elliptic problem at each time step [11,17].

Another way of approximation of gravitational terms is explicit. For example, forward-backward time differencing gives:

$$
\begin{gathered}
\frac{\hat{u}^{n+1}-u^{n}}{\tau}=f_{0} \frac{\hat{v}^{n+1}+v^{n}}{2}-\hat{G}_{x}^{n+1}+N_{u}^{n+1 / 2}, \\
\frac{\hat{v}^{n+1}-v^{n}}{\tau}=-f_{0} \frac{\hat{u}^{n+1}+u^{n}}{2}-\hat{G}_{y}^{n+1}+N_{v}^{n+1 / 2}, \\
\frac{\hat{P}^{n+1}-P^{n}}{\tau}=-D^{n}-\dot{\sigma}_{\sigma}^{n}, \frac{\hat{T}^{n+1}-T^{n}}{\tau}=\frac{R T_{0}}{c_{p}}\left(\frac{\hat{P}^{n+1}-P^{n}}{\tau}+\frac{\dot{\sigma}^{n}}{\sigma}\right)+N_{T}^{n+1 / 2} .
\end{gathered}
$$

This scheme is of the first order of accuracy and its CFL condition is $\tau \leq \sqrt{2} h_{g} / c_{\text {grav }}$, where $h_{g}$ is meshsize used for approximation of gravitational 
waves and $c_{\text {grav }} \approx 350 \mathrm{~m} / \mathrm{s}$ is the propagation velocity of the fastest gravitational waves. This condition implies the use of the small time steps about 3 min on a fine horizontal grid with $h_{g}=50 \mathrm{~km}$. However, the algorithm of solution at each time step is very simple because this scheme is actually explicit.

Finally, let us consider implicit time differencing for essential fast gravitational waves and explicit one for secondary slow waves. To this end, we should apply vertical transform to separate the different types of the gravitational waves. First we eliminate functions $P, T$ and $\dot{\sigma}$ from (6) to obtain prognostic equation for $G$ :

$$
c_{p}\left(\sigma G_{t \ln \sigma}\right)_{\sigma}=R^{2} T_{0} D-c_{p} R\left(\sigma N_{T}\right)_{\sigma} .
$$

Then, we use the vertical expansion of the functions

$$
\varphi=\sum \varphi_{k} S_{k},
$$

where $S_{k}(\sigma)$ are the first $K$ eigenfunctions of the differential vertical structure equation

$$
c_{p}\left(\sigma_{\ln \sigma}\right)_{\sigma}=-\lambda^{-1} R S
$$

or its discrete analogues, that is, the eigenvectors of the difference vertical structure equation on vertical $K$-level grid. Using (14) we can rewrite equations (5), (13) in the following form for each vertical mode $k$

$$
u_{t k}=f_{0} v_{k}-G_{x_{k}}+N_{u k}, v_{t k}=-f_{0} u_{k}-G_{y_{k}}+N_{v_{k}}, G_{t k}=-c_{k}^{2} D_{k}+N_{G_{k}},
$$

where $c_{k}=\sqrt{R T_{0} \lambda_{k}}$ and $N_{G}=\lambda_{k} R\left(\left(\sigma N_{T}\right)_{\sigma}\right)_{k}$. It was shown in [5] for differential vertical structure equation and in [4] for difference equation that all eigenvalues $\lambda_{k}$ are positive and have zero limit point as $k$ approaches infinity. Fig. 1 shows the values of $c_{k}$ as function of the mode number $k$ for 20-level Lorenz staggered vertical grid. The results for homogeneous grid and actual grid (with concentration of the levels in boundary layer and higher troposphere) are presented.

Now we can apply different approximation to the fast and slow gravitational waves. The first $I$ principal vertical modes are approximated implicitly with second order of accuracy

$$
\begin{gathered}
\frac{u_{k}^{n+1}-u_{k}^{n}}{\tau}=f_{0} \frac{v_{k}^{n+1}+v_{k}^{n}}{2}-\frac{G_{x}^{n+1}{ }_{k}+G_{x_{k}}^{n}}{2}+N_{u}^{n+1 / 2}, \\
\frac{v_{k}^{n+1}-v_{k}^{n}}{\tau}=-f_{0} \frac{u_{k}^{n+1}+u_{k}^{n}}{2}-\frac{G_{y}^{n+1}{ }_{k}+G_{y_{k}}^{n}}{2}+N_{v}^{n+1 / 2}{ }_{k}, \\
\frac{G_{k}^{n+1}-G_{k}^{n}}{\tau}=-c_{k}^{2} \frac{D_{k}^{n+1}+D_{k}^{n}}{2}+N_{G}^{n+1 / 2}{ }_{k}, k=1, \ldots, I .
\end{gathered}
$$




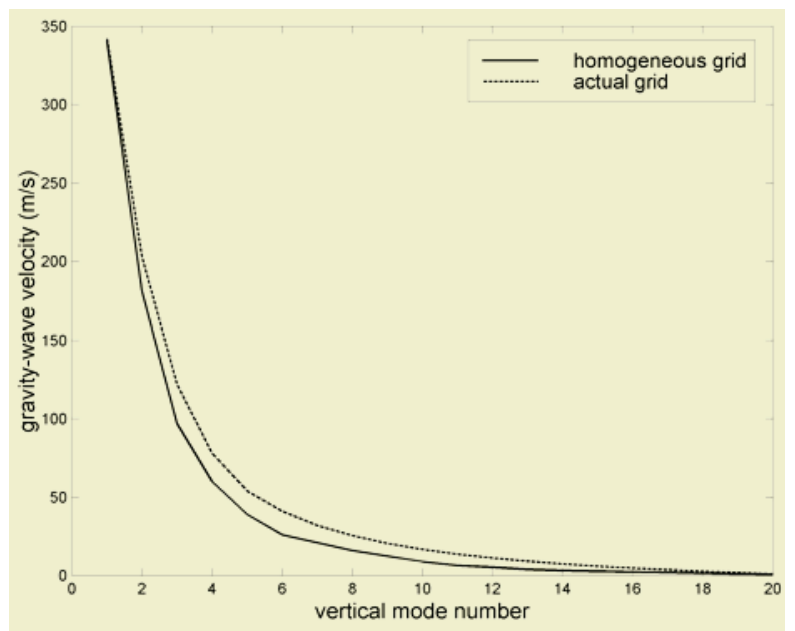

Fig. 1. Gravity-wave velocity as function of the vertical mode number

The remaining secondary modes are approximated explicitly with the first order of accuracy:

$$
\begin{gathered}
\frac{u_{k}^{n+1}-u_{k}^{n}}{\tau}=f_{0} \frac{v_{k}^{n+1}+v_{k}^{n}}{2}-G_{x}^{n+1}{ }_{k}+N_{u}^{n+1 / 2}, \\
\frac{v_{k}^{n+1}-v_{k}^{n}}{\tau}=-f_{0} \frac{u_{k}^{n+1}+u_{k}^{n}}{2}-G_{y}^{n+1}+N_{v}^{n+1 / 2}, \\
\frac{G_{k}^{n+1}-G_{k}^{n}}{\tau}=-c_{k}^{2} D_{k}^{n}+N_{G}^{n+1 / 2}{ }_{k}, k=I+1, \ldots K .
\end{gathered}
$$

Let us note that although systems (15) are coupled through nonlinear terms, both schemes (16)-(18) and (19)-(21) can be solved separately for each $k$ because nonlinear terms are treated explicitly. This approach generates stability condition in the form $\tau \leq \sqrt{2} h_{g} / c_{I+1}$, where $c_{I+1}$ is the maximum gravity-wave speed of the modes treated explicitly.

If discrete vertical transform is applied, implicit approximation (16)-(18) of all the vertical modes will result in the scheme (7)-(9) and explicit approximation (19)-(21) of all the modes will give forward-backward scheme (10)-(12).

\section{Numerical Experiments}

In this section we present the results of the experiments with different configurations of the vertical approximation. At each time step, the explicit approximation (19)-(21) is solved by direct formulas: first $G_{k}^{n+1}$ are found from (21) and then $u_{k}^{n+1}$ and $v_{k}^{n+1}$ 
are elementary solved from (19) and (20). The implicit approximation (16)-(18) is reduced to $2 \mathrm{D}$ Helmholtz equation for $G_{k}^{n+1}$ :

$$
\nabla^{2} G_{k}^{n+1}-\frac{4+\tau^{2} f_{0}^{2}}{\tau^{2} c_{k}^{2}} G_{k}^{n+1}=F_{k}^{n},
$$

where ritght-hand side is combination of the values at the time level $t_{n}$. This equation is solved by multigrid method, which is fast solver for such kind of the problems. Its optimal versions require $O(M N)$ arithmetic operations, where $M$ and $N$ are the number of points in horizontal. We apply BOXMG algorithm [2,6] based on Galerkin type of discretization, which allows to use spatial grids with arbitrary number of the points with no less of the convergence speed of iterations. Numerical experiments showed that optimal version of the BOXMG algorithm for equation (22) consists of using the $\mathrm{V}$-cyclic method with two cycles for the first two vertical modes and one cycle for others. One four-color Gauss-Seidel point relaxation sweep is performed on any grid both before dropping down to the next coarser grid and before interpolation to the previous finer grid. As initial guess for iterations we use $G_{k}^{n}$. After $G_{k}^{n+1}$ is found, the $u_{k}^{n+1}$ and $v_{k}^{n+1}$ are calculated by elementary solution of (16), (17).

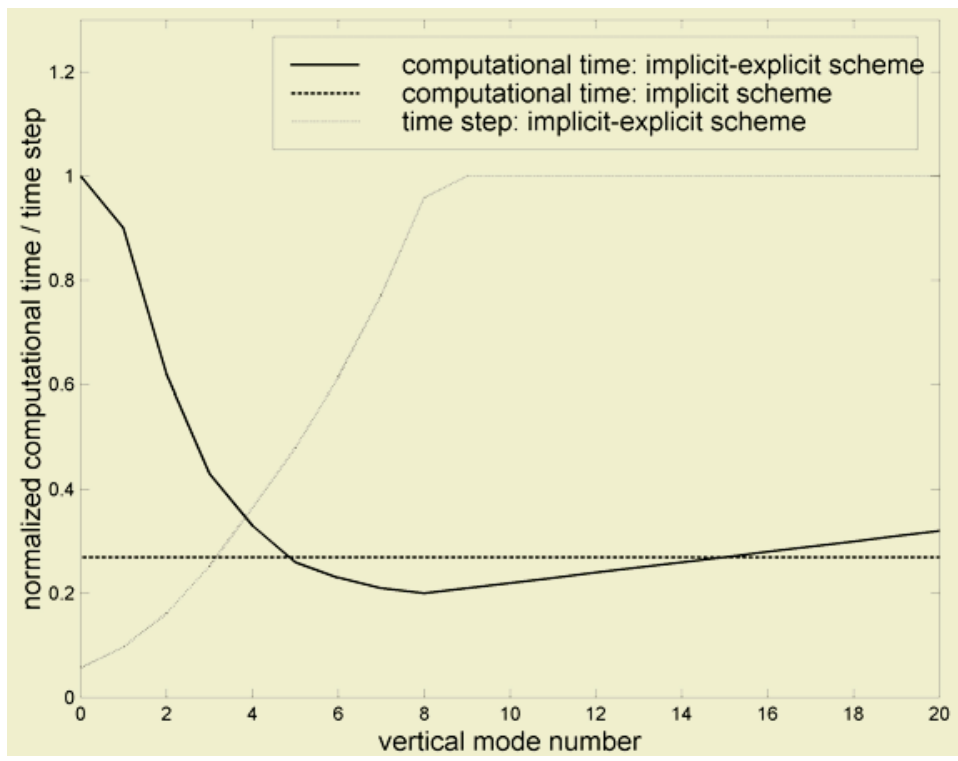

Fig. 2. Time step and computational cost of the implicit-explicit schemes

24-hour integrations of the primitive equations were carried out for different number of the vertical modes approximated implicitly. Fig. 2 shows time step used in these experiments (chosen in accordance with stability criterions) and computational time cost (in percent of the forecast time of the forward-backward scheme (10)-(12)) 
as functions of the number of the implicitly treated vertical modes. The required computational time for one forecast computed by implicit approximation (7)-(9) is also shown. The last integration is less expensive than that with implicit solution of all vertical modes by formulas (16)-(18). The former requires solution of the 3D elliptic equation but does not need application of vertical tranform, while the latter uses vertical transform and fast $\mathrm{MG}$ solvers for a set of $2 \mathrm{D}$ elliptic problems. Although multigrid solution of $3 \mathrm{D}$ equation is more expensive than fast solution of the decoupled set of 2D equations, the additional computational charge due to vertical transform is too hard. Since both schemes have absolutely stable adjustment step, that is, their maximum time step is about $60 \mathrm{~min}$ as defined by advective step, the scheme (7)-(9) required less computational time than (16)-(18) with $I=K$. Nevertheless, some versions of the implicit-explicit algorithm are more computationally efficient than the standard algorithm (7)-(9). For example, implicit treatment of 7-9 vertical modes gives certainly more efficient algorithm.

To evaluate forecasting ability of the above schemes we carried out integrations based on actual atmospheric data. The horizontal domain of $5000 \times 5000 \mathrm{~km}^{2}$ centered at Porto Alegre city $\left(30^{\circ} \mathrm{S}, 52^{0} \mathrm{~W}\right)$ was covered by uniform spatial grid $C$ with meshsize $h=50 \mathrm{~km}$ (we use Arakawa-Mesinger nomenclature of spatial grids [12]). The initial and boundary conditions were obtained from objective analysis and global forecasts of National Centers for Environmental Prediction (NCEP).

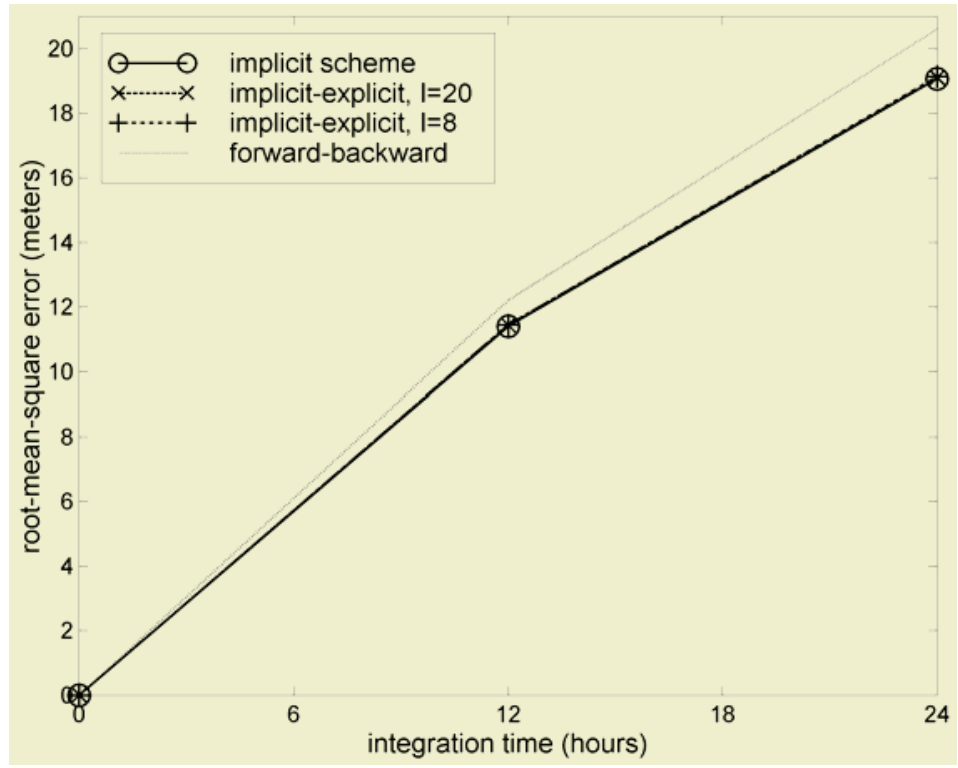

Fig. 3. Root-mean-square error of geopotential forecast at the surface of $500 \mathrm{hPa}$

It is well expected that after certain period of integration the forecast results will be determined in high degree by boundary conditions. Therefore the standard evaluation time for the regional models is limited to 24 or 36 hours of integration [1]. In Fig. 3 
we present the root-mean-square differences in meters between 24-h forecasts and analysis at pressure level $500 \mathrm{hPa}$. This is standard measure of quality of short-range weather forecasts for geopotential fields [1]. Each scheme was run with appropriate time step chosen in accordance with stability criterions. Based on this results we can conclude that the most efficient algorithms are obtained when implicit-explicit algorithm with 7-9 implicit modes is used.

\section{Acknowledgements}

This research was supported by brazilian science foundation $\mathrm{CNPq}$ under grant 302738/2003-7.

\section{References}

1. Anthes R.A., Kuo Y.H., Hsie E.Y., Low-Nam S., Bettge T.W.: Estimation of skill and uncertainty in regional numerical models. Q. J. R. Meteorol. Soc. 115 (1989) 763-806.

2. Bandy V., Sweet R.: A set of three drivers for BOXMG: a black box multigrid solver. Comm. Appl. Num. Methods 8 (1992) 563-571.

3. Bourchtein A.: Semi-Lagrangian semi-implicit space splitting regional baroclinic atmospheric model. Appl. Numer. Math. 41 (2002) 307-326.

4. Bourchtein A., Kadychnikov V.: Well-posedness of the initial value problem for vertically discretized hydrostatic equations. SIAM J. Num. An. 41 (2003) 195-207.

5. Cohn S.E., Dee D.P.: An analysis of the vertical structure equation for arbitrary thermal profiles. Q. J. R. Meteorol. Soc. 115 (1989) 143-171.

6. Dendy J.E.: Black box multigrid. J.Comp.Phys. 48 (1982) 366-386.

7. Durran D.: Numerical Methods for Wave Equations in Geophysical Fluid Dynamics. Springer, New York (1999).

8. Holton J.R.: An Introduction to Dynamic Meteorology. Academic Press, San Diego (1992).

9. Hortal M.: The development and testing of a new two-time-level semi-Lagrangian scheme (SETTLS) in the ECMWF forecast model, Q.J.R.Met.Soc. 128 (2002) 1671-1687.

10. Mcdonald A.: A semi-Lagrangian and semi-implicit two time level integration scheme. Mon. Wea. Rev. 114 (1986) 824-830.

11. McDonald A., Haugen J.: A two-time-level, three-dimensional semi-Lagrangian, semiimplicit, limited-area gridpoint model of the primitive equations. Mon. Wea. Rev. 120 (1992) 2603-2621.

12. Mesinger F., Arakawa A.: Numerical Methods Used in Atmospheric Models. GARP Publ. Ser. 17(I), Geneva (1976).

13. Pudykiewicz J., Benoit R., Staniforth A.: Preliminary results from a partial LRTAP model based on an existing meteorological forecast model. Atmos.-Ocean 23 (1985) 267-303.

14. Robert A., Yee T.L., Ritchie H.: A semi-Lagrangian and semi-implicit numerical integration scheme for multilevel atmospheric models. Mon. Wea. Rev. 113 (1985) 388-394.

15. Staniforth A., Côté J.: Semi-Lagrangian integration schemes for atmospheric models - A review. Mon. Wea. Rev. 119 (1991) 2206-2223.

16. Temperton C., Staniforth A.: An efficient two-time-level semi-Lagrangian semi-implicit integration scheme, Q. J. R. Meteorol..Soc. 113 (1987) 1025-1039.

17. Temperton C., Hortal M., Simmons A.J.: A two-time-level semi-Lagrangian global spectral model. Q. J. R. Meteorol. Soc. 127 (2001) 111-126. 